This text was published in Bühler-Dietrich, Annette et al. (eds.), Topos Tier : neue Gestaltungen des Tier-Mensch-Verhältnisses, transcript Bielefeld, 2016.

It is posted here by permission of transcript Verlag for personal use only, not for redistribution. http://www.transcript-verlag.de/978-3-8376-2860-9/topos-tier

\title{
Experimentalobjekte. Tiere als Figuren anthropologischen Wissens
}

BENJAMIN BÜHLER

Wegen der konstitutiven und keineswegs komplikationslosen Unterscheidung von Tier und Mensch lässt sich die Geschichte des Menschen auch als eine Geschichte des Tieres lesen, das heißt: als Geschichte der Operationen, die die Unterscheidung von Tier und Mensch herstellen und in denen gleichwohl das Tier als das ausgeschlossene Element auf der Seite des Menschen wiederkehrt. Die Grenze zwischen Tier und Mensch ist dabei nicht eine einfache singuläre Linie, weshalb es nicht ausreicht, alleine die Kriterien aufzulisten, durch welche der Mensch vom Tier unterschieden wird und welche möglicherweise im Laufe der Geschichte ihre Gültigkeit verlieren - weil Tiere eben doch über Sprachen verfügen, doch denken, lügen und täuschen. $\mathrm{Zu}$ untersuchen ist vielmehr der durch den Unterschied von Tier und Mensch aufgespannte Grenzraum, innerhalb dessen die Unterscheidung Tier/Mensch mit all ihren Grenzfiguren allererst hergestellt wird, wie es Jacques Derrida mit seinem Begriff "limitrophy“ vorgeschlagen hat. ${ }^{1}$

1 „Limitrophy is therefore my subject. Not just because it will concern what sprouts or grows at the limit, around the limit, by maintaining the limit, but also what feeds the limit, generates it, raises it, and complicates it. Whatever I will say is designed, certainly not to efface the limit, but to multiply its figures, to complicate, thicken, delinearize, fold, and divide the line precisely by making it increase and multiply." Derrida, Jacques, „The Animal That Therefore I Am (More to Follow)“, in: Critical Inquiry 28, Winter (2002), S. 369-418, Zitat: S. 397f. (zuerst erschienen unter dem Titel: „L'animal que donc je suis (à suivre)“, in Mallet, Marie-Louise (Hg.), L'Animal autobiographique. Autour de Jacques Derrida, Paris: éditions Galilée 1999, S. 251-301). 
Die Wiederkehr des Tiers auf der privilegierten Seite der Unterscheidung erfolgt dabei zunächst auf einer technischen Ebene, wenn etwa Praktiken aus dem Tierbereich auf den menschlichen Bereich übertragen werden. Und auf der epistemischen Ebene kehrt das Tier insofern wieder, als dass ein bestimmtes Wissen vom Menschen alleine über den Umweg über das Tier gewonnen werden kann. So werden Tiere in Experimentalsystemen zu Objekten des Wissens und fungieren dabei als Substitute des Menschen, wie im Folgenden zunächst an der Geschichte von Tieren als Experimentalobjekten ${ }^{2}$ und dann am Beispiel der Experimentalisierung des Verhaltens um 1900 aufgezeigt werden soll.

\section{SUBSTITUTE IN DER ANATOMIE}

Wer etwas über den Menschen, seine Anatomie, seine Physiologie oder Pathologie erfahren will, muss den Menschen selbst zum Objekt der Untersuchung machen, und zwar am besten den lebenden Menschen. Ganz in diesem Sinne handelten die beiden griechischen Ärzte Herophilus und Erasistratus im 3. Jh v. Z. Von beiden sind zwar keine vollständigen Schriften erhalten, dennoch wurden sie bis in das 20. Jahrhundert zitiert als Autoritäten in Sachen Vivisektion. Über ihr Vorgehen schreibt der römische Gelehrte Aulus Cornelius Celsus, die Position von Vertretern einer theoretischen Medizin wiedergebend:

Wenn [...] in den inneren Teilen des Köpers Schmerzen und verschiedene Arten von Krankheiten entständen, so könne (ihrer Meinung nach) niemand auf Teile, die ihm selbst unbekannt sind, die richtigen Mittel anwenden: deshalb sei es notwendig, tote Körper zu öfnen und deren Inneres zu untersuchen. Ganz vorziglich hatten daher Herophilus und Erasistratus gehandelt, indem sie Verbrecher, welche sie von den Königen aus den Gefängnissen emptingen, lebend offneten und so, während sogar das Atmen noch fortbestand, die Teile betrachteten, welche die Natur vorher dem Auge entzogen hatte [... . ${ }^{3}$

2 Einen historischen Uberblick hierzu bieten z.B. Guerrini, Anita, Experimenting with Humans and Animals, From Galen to Animal Rights, Baltimore: John Hopkins University Press 2003; Maehle, Andreas-Holger, Kritik und Verteidigung des Tierversuchs, Die Anfänge der Diskussion im 17. und 18. Jahrhundert, Stuttgart: Franz Steiner Verlag 1992; Bongards, Roland/Pethes, Nicolas (Hg.), Tier-Experiment-Literatur 1880-2010, Würzburg: Königshausen und Neumann 2013.

3 Celsus, Aulus Comelius, Über die Arzneiwissenschaft in acht Büchern, Hildesheim: Olms 1967, S. 24. 
Moral spielt hier keine Rolle. Nach Celsus sei es nicht, wie viele meinten, grausam, wenn man durch Aufopferung von noch dazu wenigen Verbrechern für die rechtschaffenden Menschen aller Jahrhunderte Heilmittel zu erforschen strebe. Allerdings führt Celsus auch die Gegenposition an: So würden Ärzte die Sektionen bei lebendigem Leib ausdrücklich als überflüssig und grausam verdammen. Auch diese Ärzte führen kein moralisches, sondern ein wissenschaftliches Argument an. Denn wenn ein Körper aufgeschnitten werde, würden durch Furcht und Schmerz der aufgeschnittenen Menschen sowie durch das Schneiden und die zugefügten Wunden die inneren Teile derartig geschädigt, dass die Erkenntnisse nur falsch sein könnten. Nichts seit törichter, so beschreibt Celsus diese Position, als der Wahn, im lebenden Körper seien die Teile ebenso beschaffen wie im sterbenden oder gar toten Körper.

Ist daher schon die Sektion des lebenden und/oder toten menschlichen Körpers problematisch, weil jeder Eingriff das verändert, was beobachtet werden soll, steigern sich die Schwierigkeiten dann, wenn Menschen weder bei lebendigem noch bei totem Leib aufgeschnitten werden dürfen, womit der römische Arzt Galen konfrontiert war. Die Sektion menschlicher Körper war generell in Rom zur Lebenszeit Galens verboten. Anschaulich beschreibt der Anatom Rufus von Ephesus aus dem 2. Jh. diese Situation, der während einer Sektion seinen Zuschauern zugerufen haben soll:

Listen then, and look at this slave, and you shall commit to memory first what is superficially visible. Next I will try to teach you what the interior parts are to be called by dissecting some animal which is most like a human being. For, even if they are not alike in every respect, still there is nothing to prevent one from demonstrating at least the essentials of every part. In the old days these matters were demonstrated in a more noble fashion, on the human subject. ${ }^{4}$

Da man nicht mehr wie Herophilus und Erasistratos Menschen bei lebendigem Leib aufschneiden durfte, sondern Menschen (bzw. Sklaven) nur als Beobachtungsobjekte zur Verfügung standen, ging der Anatom zu den Tieren über. Dieses Vorgehen hat Galen auch theoretisch gefasst, indem er die für die Sektion verwendeten Tiere nach dem Kriterium ihrer Ähnlichkeit mit dem Menschen betrachtete: An erster Stelle stehen die Affen, allen voran die dem Menschen ähnlichsten Affen, danach folgen alle die, denen man den Namen „Tier" geben kön-

4 Zit nach: May, Margaret T., „Introduction“, in: Galen, On the Usefulness of the Parts of the body [De usu partium], hg. und übers. von Margaret T. May, Ithaca/New York: Cornell University Press 1968, S. 24. 
ne: „Of these first all that form the ape-like class, and after these bears, and then next the carnivores, then mice and theirs kind, and then the so-called, wholehooved" animals, and sixthly, the ruminants. ${ }^{.5}$ Galen selbst sezierte vor allem Affen, aber durchaus auch Schweine und mindestens einen Elefanten. Zwar durfte er keine Menschen sezieren, doch menschliche Skelette standen ihm zur Verfügung, beispielsweise aufgefundene, von Aasfressern bis auf die Knochen abgenagte Leichen. Galen schrieb also durchaus, dass er hauptsächlich durch Affen sein Wissen über Anatomie gewann, der Übertragungsproblematik widmete er sich in seinem Werk De Anatomicis Administrationibus. Dagegen war in seinem großen Werk über die Anatomie und Physiologie des Menschen De usu partium diese Genealogie nicht mehr sichtbar. Dieses Buch aber wurde zu einer der zentralen Quellen der frühneuzeitlichen Medizin.

Dass Galen die menschliche Anatomie aus der Übertragung von Sektionen an Tieren entwickelte, blieb unter anderem deshalb weitgehend verborgen, weil die Forscher ihr Wissen vor allem aus der Lektüre seiner (und anderer) Werke gewannen, selbst aber keine oder zumindest keine systematischen Sektionen vornahmen. Dies sollte sich erst im 16. Jahrhundert ändern, wegweisend für die neue, auf Erfahrung gegründete Anatomie wurde das Werk De humani corporis fabrica aus dem Jahr 1543 des italienischen Anatomen Andreas Vesalius. So zeigt das Titelbild des Buches nicht nur, wie Vesalius vor Publikum eine menschliche Leiche seziert, sondern auch, dass bereits ein Hund und ein Affe für ihre Sektion bereitstehen (Abb. 1). Sämtliche Schriften, die er gelesen habe, so Vesalius in der Vorrede zu seinem Werk, bezögen ihr Wissen über die Anatomie des Menschen aus Galens Werken, welche sie für völlig fehlerfrei hielten. Nun wisse man aber, dass Galen niemals selbst einen menschlichen Körper seziert habe, sondern, bis auf wenige Ausnahmen, den Bau des menschlichen Körpers aus der Sektion von Affen abgeleitet habe und dabei die Differenz zwischen den Organen von Affen und Mensch mehrmals missachtet habe.

Der Wandel der frühneuzeitlichen Anatomie resultiert damit aus einer doppelten Wendung: Erstens sollen die Forscher nicht mehr nur Bücher lesen, sondern selbst Körper aufschneiden. Und zweitens müsse man Menschen sezieren, denn Übertragungen vom Tier auf den Menschen führten zu falschen Schlüssen. Dabei war auch im 16. Jahrhundert der Bezug von Leichen nicht einfach: Vesalius bezog seine Leichen von Hinrichtungen, und angeblich soll er seine Studenten angewiesen haben, des Nachts Leichen aus Gräbern zu stehlen. Zum Teil

5 Galen, On Anatomical Procedures [De Anatomicis Administrationibus], hg. und tibers, von Charles Singer, London u.a. 1956, S. 153. 
zeigt sich die Herkunft der Untersuchungsobjekte noch in den Abbildungen (Abb. 2).

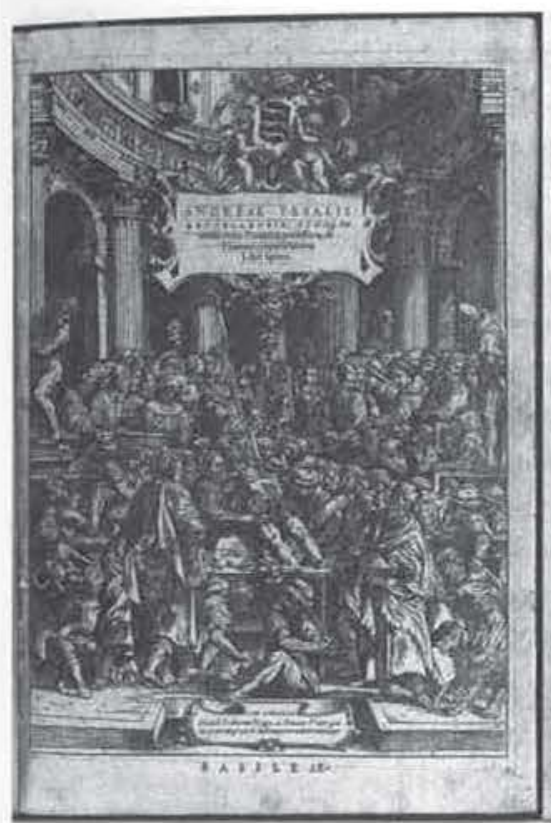

Abb, 1: Andreas Vesalius, Suorum de humani corporis fabrica librorum epitome, Basileae 1543.

Quelle: Andreas Vesalius, Stortum de humani corporis fabrica librorum epitome, Basileae 1543: Wellcome Library, London, L0046304,

http://wellcomeimages,org/ (Datum des letzten Zugriffs: 05.06.2014).

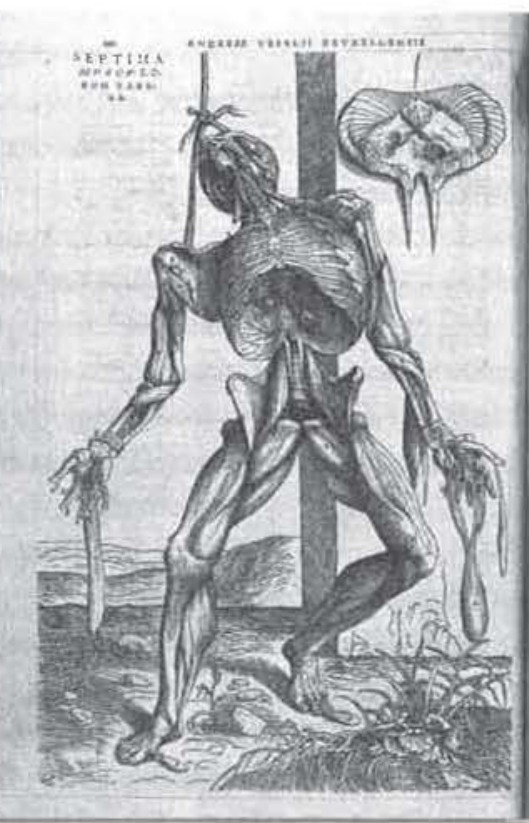

Abb. 2: An einem Galgenstrick hängendes Skelett nach Andreas Vesalius, Suorum de humani corporis fabrica librorum epitome, Basileae 1543, S. 190.

Quelle: Wie Abb. 1

\section{SUbstitute in DER fRühneUzeitlichen Physiologie}

Der Frage nach dem Untersuchungsobjekt kam somit eine zentrale Bedeutung zu. Dabei verschärfte sich die Situation für die Forscher dadurch, dass zwar die Anatomie auf Leichen rekurrieren konnte, sobald sich aber das Interesse auf die 
Funktionen von Körperteilen, zum Beispiel die Funktion der Blutgefäße richtete, stand man wieder vor demselben Problem. Denn auch wenn man menschliche Leichen aufschneiden konnte, Experimente am lebenden Menschen unterlagen einem Tabu. So verurteilte etwa der zu Beginn des 17. Jahrhunderts berühmte französische Anatom Jean Riolan ausdrücklich die Menschenvivisektionen von Herophilus und Erasistratos und verdächtigte sogar Vesalius, solche Versuche vorgenommen zu haben. Tierversuche jedoch akzeptierte Riolan. In seinem Werk Schola anatomica (1608) schrieb er: ,Es ist besser, nach dem Beispiel Galens die Vivisektion an Tieren durchzuführen, an denen man dasselbe wie am lebenden Menschen feststellen und beobachten kann."

Die Möglichkeit, Tiere bei lebendigem Leib aufzuschneiden und dann auch Experimente an ihnen durchzuführen, wurde solchermaßen zum Forschungsprogramm der Lebenswissenschaften: Francis Bacon erkannte in seiner Wissenschaftsutopie Nova Atlantis (1627) in den Tieren bedeutende Versuchsobjekte: In dem als Paradigma einer zukünftigen Wissenschaft stehenden Haus Salomons' führt man an Tieren Sektionen und chirurgische Verfahren durch, um Aufklärung über den menschlichen Körper zu erhalten, Versuche mit Giften sollen den menschlichen Körper widerstandsfähiger machen, und die Kreuzung verschiedener Tiere solle vollkommen neue Arten hervorbringen. In Bacons Vision wird das Tier zum allzeit verfügbaren Versuchsobjekt, als welches es nicht zuletzt die Erkenntnis des Menschen selbst ermöglicht. Praktisch umgesetzt findet sich dieses Programm dann bei William Harvey, der mit seinen Untersuchungen über den Blutkreislauf der Physiologie als Experimentalwissenschaft den Weg wies:

When I first gave my mind to vivisections, as a means of discovering the motions and uses of the heart, and sought to discover these from actual inspection, and not from the writings of others, I found the task so truly arduous, so full of difficulties, that $I$ was almost tempted to think [... that the motion of the heart was only to be comprehended by God. ${ }^{7}$

Wie Harvey dann aber zeigt, kann der Forscher durch Ausdauer und eine möglichst große Anzahl von Vivisektionen und Beobachtungen an einer möglichst großen Vielfalt von Tieren die Perspektive Gottes einnehmen und die Bewegungen des Blutes und des Herzens beschreiben: Harveys streng experimentelles

6 Zit, nach: Maehle, Kritik und Verteidigung des Tierversuchs, (Ann. 2), S. 72.

7 Harvey, William, ,An anatomical disquisition on the motion of the heart and blood in animals", in: The Works of William Harvey, übers, von Robert Willis, mit einer Einl. von Arthur C. Guyton, Philadelphia 1989, S. 1-86, Zitat: S. 19. 
Vorgehen sollte zum Paradigma der experimentalwissenschaftlichen Ausrichtung der Lebenswissenschaften werden. ${ }^{8}$ Dabei blieb die Antike als Argumentationsfolie auch weiterhin erhalten, wie Robert Boyles Ausführungen zu Tieren als Experimentalobjekten, in denen die Namen der beiden griechischen Ärzte einmal mehr eine nicht zu überschreitende Linie markieren, zeigen:

[...] the experience of our own age may suffice to manifest, what light the anatomical doctrine of man's body may receive from experiments made on other subjects. For since it were too barbarous, and too great a violation of the laws, not only of divinity but humanity, to dissect human bodies alive, as did Herophilus and Erasistratus, who [...] obtained of kings the bodies of malefactors for that purpose, and scrupled not to destroy man to know him; and since nevertheless, divers things in anatomy, as particular the motion of the blood and chyle cannot be discovered in a dead dissected body [...] that may be seen in one opened alive; it must be very advantageous to a physician's anatomical knowledge, to see the dissections of dogs, swine, and other living creatures, made by an inquisitive naturalist. $^{9}$

Boyles Beispiele lesen sich wie ein Katalog der zeitgenössischen Forschungen und beleuchten die Frage, wie gerade die Beobachtungen an Tieren gängige Positionen zur Revision zwingen oder in Frage stellen. So überlebten Hühner oder Schildkröten die Entfernung des Kopfes, woraus folge, dass die Doktrin der Notwendigkeit eines unaufhörlichen Einflusses des Gehirns auf den Körper überdacht werden müsse. Die Regeneration von Körpergliedern nach deren gewaltsamer Entfernung bei Eidechsen oder Krebsen stelle die Lehrmeinung in Frage, dass der Verlust solcher Glieder irreparabel sei. Experimente könnten aber auch Lehrmeinungen bestätigen. So stellte Boyle selbst Versuche zur Ver-

8 Harvey vergleicht die Embryonen verschiedener Tiere mit denen des Menschen und stellt fest, dass die Abläufe bei gewissen tierischen und menschlichen Embryonen gleich seien. Überhaupt führen ihn seine Vivisektionen durch das gesamte Tierreich, Schritt für Schritt, von den Zoophyten, die kein Herz haben bzw, deren ganzer Körper als solches fungiert, über Insekten, blutlose und kalte Tiere, wie Fische, Frösche oder Schlangen, und blutführende warme Tiere, vorzugsweise Hunde und Schweine, gelangt Harvey zum Menschen, womit sich der Kreis schließt: Um den Blutkreislauf des Menschen zu erforschen, muss ein Umweg gegangen werden, der selbst aber Tiere ohne Blut und Tiere ohne Lungen reicht.

9 Boyle, Robert, „Of the Usefulness of Natural Philosophy, Part. II. Sect. I: Of its Usefulness to Physick", in: Ders., The Works, Bd. 2, hg. von Thomas Birch, Hildesheim: Olms 1966, S. 67. 
dauung an, um die allgemein verbreitete Ansicht zu bestätigen, Fleisch werde im Magen durch eine Art Säure aufgelöst.

$\mathrm{Da}$ Menschenversuche aus moralischen und rechtlichen Gründen nicht annehmbar sind, schaffen die Tiere Abhilfe, wie Bacons Wissenschaftsutopie, Harveys Entdeckung des Blutkreislaufes oder Boyles Rechtfertigung von Vivisektionen vorführen. Wenn Tiere zu Experimentalobjekten gemacht werden, um Wissen über den Menschen zu generieren, so ist anthropologisches Wissen in einem zwischen Tier und Mensch eröffneten Raum zu verorten. Die Experimentalwissenschaften erzeugen eine Figur des Menschen, die das Resultat von Übertragungen ist.

\section{LEBENDE KÖRPER}

In seinem Buch Introduction à l'étude de la médicine experimentale (1865) schreibt Claude Bernard, einer der Begründer der Physiologie als Experimentalwissenschaft, die Leiche sei der seiner ,vitalen Bewegungen beraubte Organismus", weshalb sich die Physiologie mit dem lebenden Körper beschäftigen müsse. Nur über das Tierexperiment sei die Frage, was der Mensch für ein Lebewesen ist, zu beantworten. Zugleich bestimmt aber das Versuchsobjekt Tier die Grenzen der experimentellen Methode, ${ }^{10}$ denn der Physiologe und der Arzt dürften nie vergessen, dass „,ein Lebewesen einen Organismus und ein Individuum darstellt." Bernard kennt die Grenzen der Übertragung von Tieren auf Menschen: In Experimenten gewonnene Erkenntnisse könnten aufgrund der biologischen Unterschiede nicht ohne Vorbehalt von einer Tierart auf eine andere, vom Tier auf den Menschen verallgemeinert werden. Auch der Zustand des Versuchstiers, sein Entwicklungsstand, das Geschlecht oder die Jahreszeit würden sich auf die Versuche und ihre Ergebnisse auswirken.

Aber trotz all dieser Einschränkungen hält Bernard daran fest, dass für den. der gut zu experimentieren versteht, alles, was man an Tieren findet, aufschlussreich für den Menschen sein könne:

10 Ausführlich zum Tierexperiment bei Bernard: Canguilhem, Georges, La connaissance de la vie, 2. enw. Aufl., Paris: Librairie Philosophique Vrin 1975, S. 17-39. Eine Ubersetzung dieses Kapitels von Henning Schmidgen findet sich im Internet: Georges Canguilhem, „Das Experimentieren in der Tierbiologie“, unter: http://www,mpiwgberlin.mpg.de/Pre-prints/P189.PDF (letzter Zugriff: 05.10.2011).

11 Bernard, Claude, Einfiihrung in das Studium der experimentellen Medizin (Paris 1865), Leipzig: Johann Ambrosius Barth 1961, S. 129. 
Die Befunde an Tieren, sei es an den Gehim- und Rückenmarksnerven, sei es an den vasomotorischen und sekretorischen des sympathischen Systems, ebenso die Befunde am Kreislauf sind in jeder Hinsicht auf die Physiologie und Pathologie des Menschen übertragbar. Die Tierversuche mit tödlichen Stoffen oder unter schädlichen Bedingungen sind sehr nutzlich und durchaus schlüssig für die Toxikologie und Hygiene des Menschen. ${ }^{12}$

Die Übertragung von einer Art auf eine andere, sogar vom Frosch, Hund oder Affen auf den Menschen, ist dann, in jeder Hinsicht" möglich, wenn man sie als Organismen, als lebende Körper ansieht. Denn, so Bernard, die „Grundeigenschaften der Lebenselemente" sind allgemeingültig. ${ }^{13}$

Aus dem Tier als Substitut des Menschen wird solchermaßen ein lebender Körper erzeugt, den sich Tier und Mensch teilen. So lässt sich die Physiologie des 19. Jahrhunderts dort verorten, wo Michel Foucault das Überschreiten der biologischen Modernitătsschwelle festgemacht hat:

Jahrtausende hindurch ist der Mensch das geblieben, was er für Aristoteles war: ein lebendes Tier, das auch einer politischen Existenz fähig ist. Der moderne Mensch ist das Tier, in dessen Politik sein Leben als Lebewesen auf dem Spiel steht. ${ }^{14}$

In der Biopolitik ist der Mensch nicht mehr ein „Ja-aber-Tier" is sondern ein lebender Körper, an dem politische Regulierungs-Techniken ansetzen, also etwa

12 Ebd., S. 179.

13 Ebd., S. 177.

14 Foucault, Michel, Sexualität und Wahrheit 1. Der Wille zum Wissen, übersetzt von U1rich Raulff und Walter Seitter, Frankfurt a.M.: Suhrkamp 1983, S. 170 f.

15 Wie Thomas Macho („Tier", in: Wulf, Christoph, Vom Menschen. Handbuch der Historischen Anthropologie, Weinheim/Basel: Beltz 1997, S. 62-85, Zitate: S. 62f.) ausführt, ist es seit Aristoteles' Bestimmung des Menschen als einziges Tier, das sowohi zur Sprache als auch zur Staatenbildung fähig sei, üblich geworden, den Menschen als das Ja-aber-Tier zu charakterisieren: ,als kluges Tier, das arbeiten, sprechen, lemen, spielen, weinen und lachen, morden und Krieg führen kann: als geselliges und einsames Tier; als zeitliches Tier, das - losgerissen vom ,Pflock des Augenblicks'- als sorgendes und răchendes, planendes und trauemdes Tier aufzutreten vermag." Indem der Mensch durch eine differentia specifica zu allen anderen Lebewesen bestimmt wird, ergibt sich sowohl der Blick auf die Gemeinsamkeiten und damit die biologische Beschaffenheit des Menschen als auch auf die Differenz und damit die anthropologische Spezifität des Menschen. Allerdings könnten die Perspektiven von Zoologie und Anthropologie nicht auseinandergehalten werden: Metaphern und Geschichten bezeu- 
die Steuerung der Geburtenrate oder die Einführung sozialhygienischer Maßnahmen.

Die Tiere, an denen die Experimente vorgenommen und an denen das Wissen gewonnen wird, das auf den Menschen übertragen wird, sind reale Tiere, die reale Schmerzen erleiden - Bernard nannte den Frosch den „Hiob der Physiologie" - aber, und das ist hier der entscheidende Punkt, eingerückt in Experimentalsysteme transformieren sich diese Tiere in Objekte des Wissens. Der Wissenschaftshistoriker Georges Canguilhem sprach von der „Rückwirkung des Objekts des Wissens auf die Konstitution des Wissens, welches auf das Wesen dieses Objekts abzielt."16 Das Versuchsobjekt organisiert das an ihm gewonnene Wissen, durch welches es seinerseits transformiert wird: Die Tiere gehen den Menschen als „Übertiere" voran. ${ }^{17}$

\section{EXPERIMENTALISIERUNG DES VERHALTENS}

Einsatz der Verhaltensforscher zu Beginn des 20. Jahrhunderts ist die Abwendung von der Tierpsychologie, welche sich noch in recht simplen Anthropomorphišmen erging. Etwa führte Auguste Forel in dem Aufsatz Die psychischen Fähigkeiten der Ameisen (1901) aus, dass die Ameisen ein Gedächtnis besäßen, individuelle Pläne schmiedeten, Entschlüsse fassten und Gefühle zeigten. Der anthropomorphen Betrachtung der Tiere folgte die theriomorphe Betrachtung des Menschen, denn die Ameise besitze, so Forel, die sozialen Instinkte, die beim Menschen degeneriert seien und daher durch rationelle Zuchtwahl analog zur Haustierzüchtung ausgebildet werden müssten.

Dagegen suchten Wissenschaftler zu Beginn des 20. Jahrhunderts eine neue, objektive Perspektive auf das Verhalten von Tier und Mensch. Zum einen wollte man die Beschreibung des Verhaltens der Tiere auf wissenschaftliche Grundlagen stellen, zum anderen eine anthropomorphe Betrachtung des Tieres vermei-

gen, wie Tiere immer wieder neu im Rahmen eines „kulturellen Alphabets", eines "historisch spezifischen Wahınehmungshorizonts" mit Bedeutung aufgeladen werden, ob in antiken Fabeln oder in den Trickfilmen Walt Disneys, ob als Jagd-, Zucht-, Opfer-, Kriegs- oder Arbeitstiere.

16 Canguilhem, La connaissance de la vie, (Anm. 10), S. 39, zit. nach der Übersetzung von Schmidgen, (Anm, 10), S. 22.

17 Vgl. dazu. Bühler, Benjamin/Rieger, Stefan, Vom Ühertier: Ein Bestiarium des Wissens, Frankfurt a.M.: Suhrkamp 2005. 
den. ${ }^{18}$ Und auch die Technik der Vivisektion stand abermals auf dem Prüfstand. So rekapitulierte der russische Physiologe und Begründer der Reflexlehre Ivan Pawlow in dem Aufsatz „Vivisektion“ diese Technik von Herophilus bis Bernard. Auch Pawlow hebt die Notwendigkeit hervor, den lebenden Organismus zu untersuchen, die bisherigen Verfahren der Vivisektion aber erscheinen ihm ungenügend. Denn jeder Eingriff in das Tier, jede Operation könne zur Ursache von Entstellungen oder Herabsetzungen physiologischer Funktionen werden, die Schädigung eines Organs könne sich unberechenbar auf andere Organe auswirken. Insbesondere aber trage die Vivisektion zwar zur Klärung der Funktion bestimmter Teile bei, aber „wann und wie sich die Funktionen der einzelnen Teile bei normalem Gang der lebenden Maschine verketten und in welchem Ausmaß* lasse sich damit nicht herausfinden, denn schon die Versuchsanordnung sei ,unvermeidlich mit bestimmten Störungen des normalen Ablaufs der Vorgänge im Organismus verbunden". 19

Um zu „besseren“ Ergebnissen zu kommen, entwickelte Pawlow neue Techniken zur Untersuchung physiologischer Vorgänge, genannt seien hier die sogenannten chronischen Versuche mit operativem Eingriff: Damit diese Versuche zu echten Erkenntnissen führten, sollte erstens auf sterile Bedingungen geachtet werden, was in dieser Zeit nicht nur bei Tierexperimenten ein Neuheit war und die Einrichtung neuer Laborräume zur Folge hatte. Zweitens sollten die Versuche mit den Tieren erst erfolgen, wenn sie sich von dem operativen Eingriff erholt hätten, zum Beispiel der Zerstörung verschiedener Teile des Zentralen Nervensystems oder der Durchschneidung peripherer Nerven. Und nicht zuletzt erfolgte die Wahl des Versuchstieres nicht zufällig: Der Hund eignete sich nach Pawlow aus einer Reihe von Gründen, er schreibt:

Mit Bitterkeit muß man zugeben, daß das beste Haustier des Menschen, der Hund, gerade dank seiner hohen intellektuellen und moralischen Entwicklung am häufigsten ein Opfer des biologischen Experiments wird. Nur im Notfall macht man Versuche an Katzen, ungeduldigen, schreisüchtigen und böswilligen Tieren. Für chronische Versuche, bei denen das operierte Tier, nachdem es sich von der Operation erholt hat, zu langdauernden Beobachtungen dient, ist der Hund unersetzlich, ja mehr noch - im höchsten Grad rührend. Es hat den Anschein als sei er ein Teilhaber des Versuchs, der an ihm durchgeführt wird,

18 Exemplarisch sei hier genannt: Beer, Theodor/Bethe, Albrecht/Uexküll, Jakob von, "Vorschläge zu einer objektivierenden Nomenklatur in der Physiologie des Nervensystems", in: Zoologischer Anzeiger 22 (1899), S. 275-280.

19 Pawlow, Iwan P., „Vivisektion“, in: Ders., Sämtliche Werke, Bd, 4, Berlin: Akademie Verlag 1954, S. 3-17, S. 11. 
da er durch seine Verständigkeit und Bereitwilligkeit das Gelingen der Untersuchung auBerordentilich fördert. Nur ein grausamer Mensch könnte ein solches Tier später für einen anderen Versuch verwenden, der mit Leiden und Tod verbunden ist. ${ }^{20}$

Diese anthropomorphe Betrachtung des Hundes scheint im Widerspruch zu den Versuchen zu stehen, für die Pawlow sprichwörtlich geworden ist. Doch der Eindruck täuscht. Denn es ist gerade die Treue des Hundes, die ihn, im Gegensatz zur Individualität der Katze, zum geeigneten Versuchs-Objekt macht. Er ist das Tier, das den Experimentator nicht enttäuschen wird, das sich seinen Wünschen fügt, das ihm weder widerspricht noch eigene Wege geht. An den geduldigen Hunden führte Pawlow zuerst seine Versuche zu Verdauungsvorgängen durch, bald aber richtete sich sein Interesse auf die physiologischen Grundlagen der psychischen Tätigkeit, er schrieb:

Ich bin fest davon überzeugt, daß jetzt das rein physiologische Verständnis für vieles, was früher als psychische Tätigkeit bezeichnet wurde, festen Boden bekommen hat, und bei der Analyse des Verhaltens eines höheren Tieres einschließlich des Menschen ist es durchaus gerechtfertigt, sich in jeder Weise zu bemühen, die Erscheinungen rein physiologisch zu verstehen und zwar auf Grund der festgestellten physiologischen Prozesse. ${ }^{2 !}$

Für die Rückführung psychischer Erscheinungen auf physiologische Prozesse war nun eine Erscheinung zu finden, die nicht allzu komplex war und sich mit objektiven Methoden untersuchen ließ. Pawlow kam auf die sogenannte „psychische Speichelabsonderung", bei der ein Hund durch äußere Gegenstände gereizt wird, die für die Funktion der Speicheldrüse unwesentlich oder nur zufällig sind, also die klassische bedingte Konditionierung. (Abb, 3 und 4) Die beiden Bilder, welche nicht zuletzt die Evidenzerzeugung qua Schematisierung vorführen, zeigen die Experimentalsituation: Der Experimentator hält sich in einem anderen Raum auf, um sich selbst als Reizquelle auszuschließen, der Hund ist in ein Gestell eingespannt, die Speichelproduktion wird gemessen, indem der Ausführgang der Speicheldrüse durch einen operativen Eingriff nach außen verlegt wurde. Diese Grundanordnung ließ sich vielfach variieren, die Hunde sondern Speichel ab bei Erklingen einer Glocke oder bei bestimmten Lichtsignalen. Für Pawlow war diese Ausbildung bedingter Reflexe die Grundlage für eine Be-

20 Ebd,, S, 14.

21 Pawlow, Iwan, ,Antwort eines Physiologen an die Psychologen“ (1932), in: Ders., Sämtliche Werke, Bd. III, 2, Berlin: Akademie Verlag 1953, S. 404-430, S. 427. 
schreibung auch des Menschen, d.h. die menschliche Psyche ließ sich nun in den Termini der Verhaltensphysiologie fassen.

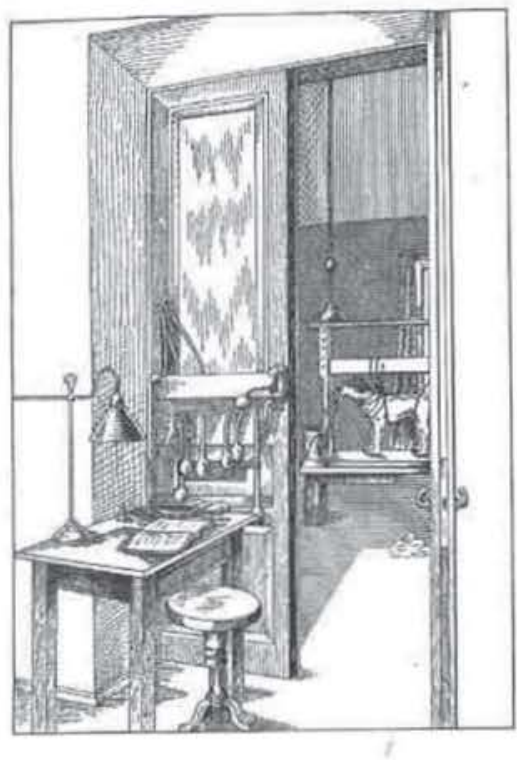

Abb. 3: Experimentalanordnung nach Pawlow 1928.

Quelle: Iwan Pawlow, „Vorlesungen uber konditionierte Reflexe (1928)", in: Ders., Sämtliche Werke, Bd. IV, Berlin 1953, S. 373.

Pawlows Reflextheorie ist die Basis einer der wichtigsten Lerntheorien des 20. Jahrhunderts, zugleich, oder gerade deshalb, aber auch Gegenstand heftiger Kritik. Dabei spielte insbesondere das Experimentalobjekt und die Schlüsse vom Hund auf den Menschen eine zentrale Rolle.

So bestreiten die Philosophischen Anthropologen Helmuth Plessner und Frederik Buytendijk in einem Aufsatz aus dem Jahr 1935 keineswegs die experimentellen Befunde Pawlows, wenden sich aber gegen die Art und Weise, mit der Pawlow aus experimentellen Daten wissenschaftliche Tatsachen macht. Pawlows Theorie sei nichts anderes als die „Übersetzung von wirklichen Be- 
obachtungen in eine imaginäre Bildersprache. ${ }^{622}$ Begriffe wie Kettenreflex oder Reflexmosaik seien Metaphern. Das Verhalten von Tieren und Menschen reduziere er auf einen Vorgang, nämlich die Speichelabsonderung bei Hunden. Ganz in diesem Sinne argumentierte auch der Psychologe Erwin Straus, der sich in seinem Buch Vom Sinn der Sinne. Ein Beitrag zur Grundlegung der Psychologie (1956) an Pawlow abarbeitete: Auch Straus bestreitet nicht die experimentellen Befunde Pawlows, wendet sich aber gegen dessen Transformation des Hundes in einen biologischen Apparat: Pawlow rede ständig von Hunden, beschreibe sie anschaulich, nenne sie mit Namen, stelle sie als Individuen dar, sie erscheinen ängstlich und scheu, angriffslustig und zutraulich. Doch wenn Pawlow von einem Hund, der hört, spreche, meine er ein Cortisches Organ, das durch Schallwellen erregt werde. Mit einem Hund, der sieht, meine er eine durch Lichtwellen erregte Netzhaut, das Essen des Hundes sei ihm keine Aktion, sondern eine Summe von Reflexbewegungen, ausgelöst durch chemische und mechanische Berührung der Mundschleimhaut. ${ }^{23}$

An der Kritik der Pawlowschen Theorie entwickeln somit Anthropologen und Psychologen eine Theorie des Experimentalobjekts, die sonst vor allem Gegenstand der historischen Epistemologie ist, die sich mit Hans Jörg Rheinberger bestimmen lässt als „Reflexion auf die historischen Bedingungen, unter denen, und die Mittel, mit denen Dinge zu Objekten des Wissens gemacht werden, an denen der Prozess der wissenschaftlichen Erkenntnisgewinnung in Gang gesetzt und in Gang gehalten wird." ${ }^{24}$ Die Objekte des Wissens sind dabei immer auch als Agenten des Wissens zu verstehen, das an ihnen gewonnen wird, wie Bruno Latour vorgeführt hat. Wird das Tier zum Gegenstand der Erfahrung, so formiert der Forscher es durch seine Operationen: Er wähit bestimmte Merkmale aus, zeichnet diese nach seinen medialen und technischen Möglichkeiten auf und kategorisiert es auf dieser Grundlage. Als Ergebnis dieser Operationen, die aus dem Tier ein Wissenschaftsobjekt machen, entsteht eine, mit Latour gesagt: unmögliche Antimomie. Denn Fakten sind einerseits durch bestimmte Operationen gemacht, andererseits taucht bei diesen Fakten etwas vom Menschen nicht Ge-

22 Buytendijk, Frederik J.J./Plessner, Helmuth, „Die physiologische Erklärung des Verhaltens. Eine Kritik an der Theorie Pawlows", in: Plessner, Helmuth, Gesammelte Schriften, Bd, 8, Frankfurt a.M.: Suhrkamp 1983, S. 7-32, S. 24.

23 Straus, Ervin, Vom Simn der Simne. Ein Beitrag zur Grundlegung der Psychologie, Reprint der 2. Aufl. von 1955, Berlin u.a.: Julius Springer 1978, S. 40.

24 Rheinberger, Hans-Jörg, Historische Epistemologie. Zw Einfithrung, Hamburg: Junius 2007, S. 11. 
machtes auf. ${ }^{25}$ Wenn in Experimentalsystemen Tiere somit zu Wissenschaftsobjekten und schließlich zu Akteuren werden, die Wissen vom Menschen erzeugen, so folgt daraus auch, dass Tier und Mensch die Plätze tauschen können.

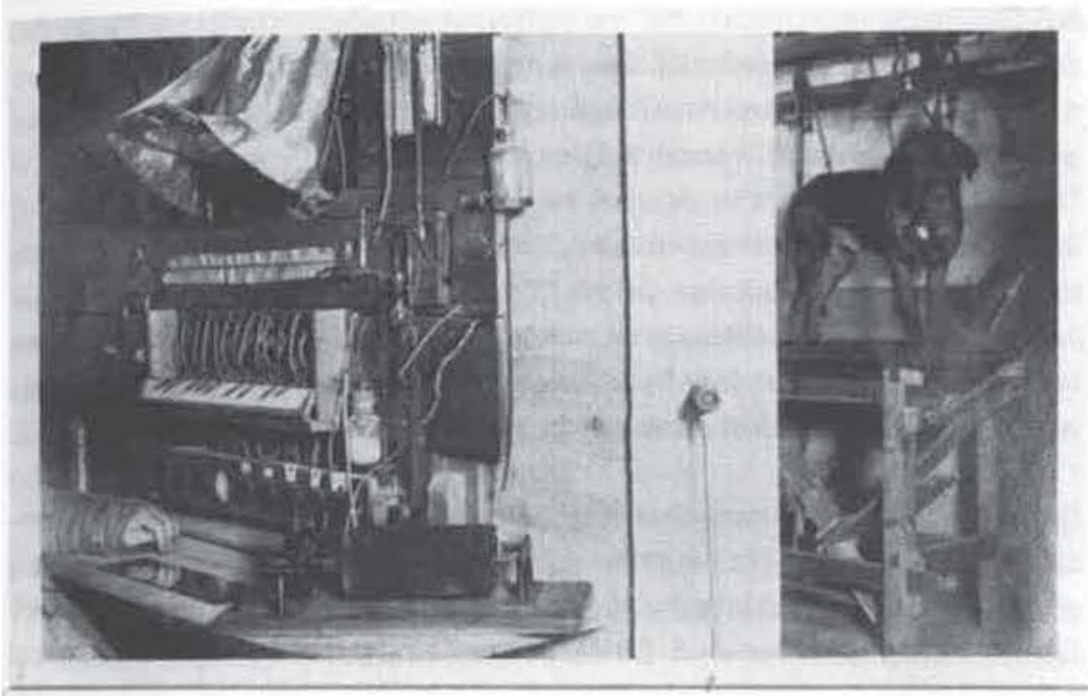

Abb. 4: Photographie aus Pawlows Labor nach Physiological Society Papers $1921 / 3$.

Quelle: Physiological Society Papers: Photographien aus I. P. Pawlows Labor (1921/3): Wellcome Library, London, L0023485, http://wellcomeimages.org/ (Datum des letzten Zugriffs: 05.06.2014).

\section{SeitenWechsel}

Besonders deutlich wird die Möglichkeit des Positionentauschs von Tier und Mensch in der amerikanischen Variante der Verhaltenstheorie, dem Behaviorismus. Das Paradeobjekt der amerikanischen Verhaltensforscher war die Ratte. $\mathrm{Ob}$

25 Latour, Bruno, Die Hoffining der Pandora, Frankfurt a. M.: Suhrkamp 2000, S. 151. Pointiert schreiben Belliger, Andrea und Krieger, David „Einführung in die AkteurNetzwerk-Theorie“, in: Dies. (Hg.), ANThology. Ein einfiuhrendes Handbuch zur Akteur-Netzwerk-Theorie, Bielefeld: transcript 2006, S. 13-50, S. 33: „Etwas ist entstanden, was vorher nicht da war, und das, was vorher da war, hat sich verăndert.“ 
Ratten durch Labyrinthe laufen, auf Knöpfe drücken müssen, um Futter zu erhalten, oder ob sie beim Drücken auf falsche Knöpfe Stromschläge erhalten - Ratten sind (neben Tauben) für die Behavioristen die bevorzugten Experimentalobjekte für das Studium von Lernvorgängen und Verhaltensweisen. Während es in der Genetik naturgemäß um die Vererbbarkeit von Eigenschaften geht, ist für den Behavioristen das Verhalten des Menschen völlig unabhängig von den genetischen Grundlagen. In einem Gedankenexperiment hat das der Begründer des Behaviorismus, John B. Watson, auf den Punkt gebracht:

Gebt mir ein Dutzend gesunder, wohlgebildeter Kinder und meine eigene Umwelt, in der ich sie erziehe, und ich garantiere, daß ich jedes nach dem Zufall auswähle und es zu einem 'Spezialisten in irgendeinem Beruf erziehe: zum Arzt, Richter, Künstler, Kaufmann oder zum Bettler und Dieb, ohne Rücksicht auf seine Begabungen, Neigungen, Făhigkeiten, Anlagen und die Herkunft seiner Vorfahren. ${ }^{26}$

Das Feld, auf dem Watson diese Theorie nicht nur behaupten, sondern experimentell auch beweisen wollte, waren die Emotionen Furcht, Wut und Liebe. Gegen den damaligen Stand der Psychologie versuchte er zu zeigen, dass auch Gefühlsreaktiônen erworben sind. Den Weg zur Klärung dieser Frage wies das zitierte Gedankenexperiment, d.h. Versuche mit Kleinkindern. Das größte Problem, das in der Kontrolle der experimentellen Bedingungen bestand, konnte gelöst werden, indem die Versuche an Kindern vorgenommen wurden, die in Heimen unter der Aufsicht der Experimentatoren aufgezogen worden waren. Einige dieser Kinder standen fast von der Geburt bis zur Vollendung des ersten Lebensjahres unter Beobachtung. In einem der berühmtesten Versuche untersuchte Watson zusammen mit Rosalie Rayner die Furchtreaktion an einem 11 Monate alten Baby, dem ,kleinen Albert". Albert hatte sein bisheriges Leben ausschließlich im Heim verbracht und war, wie Watson feststellte, ein ausgesprochen artiges Baby, das die beiden Forscher in den Monaten der gemeinsamen Arbeit nie weinen sahen, zumindest „bevor wir nicht unsere Experimente gemacht hatten. ${ }^{427}$ Die Experimentalanordnung bestand in einer klassischen Konditionierung: ${ }^{28}$ Man löste bei dem kleinen Albert eine Fluchtreaktion durch laute Geräu-

26 Watson, John B., Behaviorismus. Ergänzt durch den Aufsatz „Psychologie, wie sie der Behaviorist sieht", hg. von Carl F. Graumann, Frankfurt, a.M.: Fachbuchhandlung für Psychologie 1976, S. 123.

27 Ebd., S. 170.

28 Watson, John B./Rayner, Rosalie, „Studies in Infant Psychology“, in: The Scientific Monthly 13. 6, Dec. (1921), S. 493-515. 
sche aus, verknüpfte dann diese Reize mit einem neuen Reiz, bis am Ende allein der neue Reiz die Fluchtreaktion auslöste. Dieser neue Reiz bestand in nichts anderem als einer Ratte: Rayner und Watson nahmen eine weiße Ratte, mit der Albert schon seit Wochen gespielt und vor der er keinerlei Angst gehabt hatte, aus dem Korb heraus und hielten sie vor ihn hin. Genau dann, wenn die Hand des Jungen das Tier berührte, wurde hinter seinem Kopf auf eine Eisenstange geschlagen. Da das Kind daraufhin in einen verwirtten Zustand geriet, konnte eine Woche lang kein weiteres Experiment mit ihm durchgeführt werden. Am Ende fing der lunge bereits bei dem Anblick der Ratte zu schreien an, begab sich auf alle viere und krabbelte schnell davon. Damit war der Anfang gemacht für Versuche, die zeigen sollten, dass für die Erklärung emotionaler Reaktionen nicht auf die Vererbung als Erklärungsmuster zurückgegriffen werden musste. Der kleine Albert aber blieb vor weiteren Versuchen verschont, da er adoptiert wurde, weshalb Watson und Rayner allerdings auch nicht diese Furchtreaktion löschen konnten wie sie angeblich wollten.

Wie auch immer man die Versuche mit dem kleinen Albert beurteilen mag, sie zeigen, dass für den Behavioristen Tier und Mensch austauschbar sind, der Wechsel der Position der weißen Ratte, vom Wissenschaftsobjekt zum bedingten Reiz, führt genau diese Austauschbarkeit vor: Die Versuche am kleinen Albert sind nichts anderes als die Versuche, die zuvor mit Ratten durchgeführt worden waren, Albert wird buchstäblich zur Laborratte.

\section{LITERATUR ALS MEDIUM DES EXPERIMENTIERENS}

Während Watson später die behavioristischen Prinzipien in der Werbebranche anwendete, entwickelte der Psychologe Burrhus Skinner einen regelrechten behavioristischen Weltplan. Berühmt wurde Skinner durch seine Theorie der operationalen Konditionierung (da er nämlich im Gegensatz zu Pawlow und Watson meinte, dass die klassische Konditionierung nur sehr wenig Verhalten des Menschen erklären konnte), also das Lernen durch Belohnung, und die sogenannten Skinner-Boxen, in denen Ratten auf bestimmte Knöpfe drücken oder einen bestimmten Weg im Labyrinth zurücklegen müssen, um an Futter zu gelangen. Auch für Skinner stand die beliebige Manipulierbarkeit von Tieren und Menschen fest. So schilderte er in dem Aufsatz How to Teach Animals (1951), wie er Tauben auf Pingpong Spielen dressieren konnte, aber auch darauf, dass sie auf friedliche Artgenossen einhackten.

Was Skinner an Ratten und Tauben vorführte, sollte das Vorbild für die Organisation der menschlichen Gesellschaft bilden, Skinner entwickelte ein univer- 
sales Programm der sozialtechnischen Modellierung von Menschen. Denn nach Skinner ,,kontrollieren und steuern wir alle, und alle werden wir kontrolliert und gesteuert ". ${ }^{29}$ Nun ließen sich zwar die Experimente an einzelnen Tieren auf Lernvorgänge beim Menschen übertragen, ${ }^{30}$ doch Skinner ging es um die gesamte Gesellschaft. Da man aber mit dieser nicht experimentieren konnte und hier das Tierreich nur unzulängliche Modelle, wie den Bienen- oder Ameisenstaat, bereitstellt, griff Skinner auf ein anderes Medium zurück, nämlich die Literatur. Im Jahr 1948 veröffentlichte er den Roman Walden II, der Erstauflage folgte 1976 eine erweiterte Fassung mit Aufsätzen unter dem Titel Walden Two Revisited.

Der Übergang in die Literatur ist konsequent. Denn wie Tiere als Substitute eingesetzt werden, da Versuche am Menschen nicht erlaubt sind, so fungiert nun die Fiktion als Substitut. Was man nicht in der Realität beobachten und durchführen kann, lässt sich in Form der Erzählung sehr wohl entwerfen und bis ins Detail durchexerzieren. An die Stelle des Tierexperimentes tritt somit das Gedankenexperiment. Wie die Kulturwissenschaftler Thomas Macho und Annette Wunschel sçreiben, kämen Gedankenexperimente dann ins Spiel, wenn gar keine andere Möglichkeit bestehe, als die Konsequenzen eines Experiments in jrgendeiner Art von Erzählung zu dokumentieren und überprüfbar zu machen, anders gesagt: Wissenschaft und Literatur verbinden sich an der Stelle, an der ein Experiment nicht tatsächlich durchgeführt werden könne oder dürfe. ${ }^{31}$ Genau in diesem Sinn ist das bereits zitierte Gedankenexperiment Watsons zu verstehen, in dem er imaginierte, die soziale Umwelt der Kinder festlegen zu können, um aus ihnen dann zu machen, was er wolle. Watson setzt darüber hinaus sein

29 Skinner, Burrhus F., Wissenschaft und menschliches Verhalten, München: Kindler 1973 , S. 400.

30 „Wir untersuchen tierisches Verhalten, weil es einfacher ist. Grundlegende Prozesse können auf diese Weise leichter sichtbar gemacht und auch über größere Zeitabschnitte aufgezeichnet werden. Unsere Beobachtungen werden nicht kompliziert durch die soziale Beziehung zwischen dem Forschungsgegenstand und dem Experimentator. Bedingungen lassen sich besser kontrollieren. Wir können genetische Vorgeschichten arrangieren, um gewisse Variablen zu kontrollieren, und, um wieder andere Variablen zu kontrollieren, können wir spezielle Lebensläufe arrangieren - interessieren wir uns zum Beispiel dafür, wie ein Organismus sehen lernt, so können wir ein Tier in der Dunkelheit großziehen, bis das Experiment beginnt.“ Ebd., S. 44.

31 Wunschel, Annette/Macho, Thomas, „Mentale Versuchsanordnungen“, in: Dies. (Hg.), Science \& Fiction. Über Gedankenexperimente in Wissenschaft, Philosophie und Literatur, Frankfurt a.M.: Fischer 2004, S. 9-14, S. 11. 
Gedankenexperiment als eine regulative Fiktion ein: Es bildet den Rahmen für die Versuche mit dem kleinen Albert sowie an anderen Kleinkindern des Heimes.

Im Gegensatz zu Watsons kurzgefasstem Gedankenexperiment lieferte Skinner eine umfangreiche Utopie. Den Titel des Romans, Walden II, entlehnte er dem 1854 erschienenen Buch von Henry David Thoreau, der mit Walden das Buch schrieb, das für die Umweltbewegung der 1970er Jahre wegweisend sein sollte. Thoreau ging es um ein naturgemäßes Leben, der Mensch solle sich mit der Befriedigung seiner Grundbedürfnisse begnügen, d.h. Nahrung, Obdach, Kleidung und Heizung. In einem Selbstversuch verbrachte Thoreau zwei Jahre alleine in einer Hütte am Walden-See. Mit dem Ausdruck Walden II betreibt Skinner nun eine doppelte Erweiterung: Erstens greift er Thoreaus Ansatz auf, aktualisiert ihn aber für das 20. Jahrhundert und erweitert ihn zweitens um das Element des Sozialen: Skinners Walden ist nicht für eine Person gedacht, sondern für eine ,experimental community" ${ }^{32}$ Dabei hat er durchaus das Verhältnis von Mensch und Natur im Blick, auch wenn er in seinem Vorwort schreibt, sein Buch sei kein Handbuch fïr Hippies. Doch angesichts der Vergrößerung der Städte, der Ausbeutung der Ressourcen und der Umweltverschmutzung bräuchte es einen neuen way of life:

The choice is clear: either we do nothing and allow a miserable and probably catastrophic future to overtake us, or we use our knowledge about human behavior to create a social environment in which we shall live productive and creative lives and do so without jeopardizing the chances that those who follow us will be able to do the same. ${ }^{33}$

Auch Skinner geht es also darum, auf der Grundlage der Verhaltenstechnik eine soziale Umwelt zu erschaffen, in der Menschen sich entfalten können - und zwar tatsächlich alle Menschen. Skinner glaubte, mit der Anwendung seiner Forschungen die Zukunft der Menschheit retten zu können. Die Herkunft der dafür in Ansatz gebrachten Verhaltenstechniken verbirgt er keineswegs:

$\mathrm{Zu}$ der Zeit, als ich das Buch schrieb, hatten einige dramatische Fortschritte in experimentellen Methoden die Möglichkeit geschaffen, komplexe Verhaltensweisen [...] vorauszusagen und zu kontrollieren. Das aber betraf nur Verhaltensweisen von Ratten und Tauben. Ich vermutete zwar, daß die gleichen Methoden sich auch auf Menschen anwenden ließen,

32 Skinner, Burrhus F., „Walden II revisited“", in: Ders., Walden Two. With a new introduction by the author, Englewood Cliffs, NJ: Prentice Hall 1976, S. x.

33 Ebd., S. xvi. 
war mir dessen aber nicht sicher. Ich hatte ja noch keinerlei praktische Anwendung dieser Methoden auf den Menschen miterlebt. Die Verhaltenstechnik von Walden Two war noch ein Traum, aber der Traum sollte verwirklicht werden. Man hat inzwischen eine Technologie der Verhaltenssteuerung entwickelt, besonders für die Gebiete „Erziehung" und "Psychotherapie", und das hat direkten Bezug auf Waiden Two. ${ }^{34}$

Die Übertragung der Verhaltenstechnik von Ratten und Tauben auf Menschen kann Skinner nur in einem literarischen Gedankenexperiment durchführen, dem dann die Umsetzung in die Realität folgen sollte: Auch wenn Skinner die Wirkung seines Romans übertreiben mag, wurden tatsächlich nach dem Vorbild von Walden II experimentelle Gemeinschaften gebildet. Zum Beispiel gründeten 1973 sieben Personen, darunter einige behavioristische Psychologen, die bis heute bestehende Einrichtung Los Horcones in Mexiko mit dem Ziel, einen alternativen Lebensstil auf der Grundlage von Kooperation, Pazifismus, Gleichheit und ökologischer Nachhaltigkeit zu entwickeln, und zwar durch die Anwendung der Verhaltensforschung im Sinne Skinners. ${ }^{35}$

\section{SchlusS}

Tiere sind nicht bloße passive Objekte wissenschaftlicher Beobachtungen und Versuche, sondern immer auch Akteure. Als Experimentalobjekte sind sie reale Lebewesen, sie erleiden reale Schmerzen, und kein Konstruktivismus wird das leugnen. Indem sie jedoch eingespannt werden in wissenschaftliche Verfahrensformen, transformieren sie sich in etwas, was vorher noch nicht da war - sie werden zu Wissensfiguren. Als solche sind Tiere, ob Affe, Hund oder Ratte, ob Ameise, Fruchtfliege oder Virus, Substitute des Menschen. Und auch wenn in wissenschaftlichen Diskursen die Differenz von Tier und Mensch benannt, reflektiert und methodisch gefasst wird, wird diese Grenze doch immer wieder aufgehoben, ob die Herkunft des anthropologischen Wissens aus dem Tier ver-

34 Skinner, Burrhus, Futurum II. „,Walden Two“. Die Vision einer aggressionsfreien Gesellschaft, Reinbek bei Hamburg: Rowohlt 1974, S. 6. Dieses Vorwort hat Skinner für die deutsche Ausgabe verfasst, für die der Psychologe Werner Correll eine Einleitung schrieb. Correll war Direktor des Instituts für Programmiertes Lernen in Gießen und Verfasser des Buches Denken und Lernen. Beiträge der Lernforschung zur Methodik des Unterrichts, Braunschweig: Westermann 1971, das Skinners Aufsatz „Verhaltenspsychologische Analyse des Denkprozesses" enthielt.

35 Vgl. dazu die Homepage http://www.loshorcones.org. 
leugnet oder verdrängt wird, oder ob ein Tier und Mensch gemeinsamer lebender Körper konstruiert wird.

Die derzeitige Konjunktur der Tiere, wie etwa die Etablierung von animal studies nun auch in Deutschland zeigt, lässt sich nicht zuletzt zurückführen auf die aktuell stattindenden Verunsicherungen des Grenzverlaufs zwischen Tier, Mensch und Maschine - durch cyborgs, die Implantierung technischer Objekte in den Körper, die Herstellung transgener Tiere als Bioreaktoren oder die synthetische Biologie. Weil mit Tieren somit stets die Frage nach Grenzen, ihren Verläufen und Überschreitungen, ihren paradoxen Ein- und Ausschlussmechanismen und ihren Zwischenräumen aufkommt, sind sie der genuine Gegenstand einer Kulturwissenschaft, die sich in ihren Selbstbeschreibungen in einem Grenzoder Zwischenbereich verortet - ob die Kulturwissenschaft in einem ,offenen Prozess“" Fachgrenzen auflöst, ${ }^{36}$ Kulturforschung eine ,interdisziplinäre Arbeit an Übergängen " darstellt ${ }^{37}$ oder die Metaebene der kulturwissenschaftlichen Reflexion in einen Zwischenraum wandert, in einen ,unmarked space in between", der ,für das Herstellen bedeutsamer Beziehungen offen ist" ${ }^{\text {" }}{ }^{38}$ Die Geschichte der Tiere (sowie die der technischen Dinge) erweist sich solchermaßen als Paradigma einer gegenwärtige Problemlagen stets mitreflektierenden Kulturwissenschaft.

36 Gerbel, Christian/Musner, Lutz, „Kulturwissenschaften: Ein offener Prozess“, in: Musner, Lutz/Wunberg, Gotthart (Hg.), Kulturwissenschaften. Forschung - Praxis Positionen, Wien: WUV Universitätsverlag 2002, S. 9-23, S. 12.

37 Zentrum für Literatur- und Kulturforschung (Berlin), Europäische Kultur- und Wissenschaftsgeschichte. Forschungsprogramm 2008-2013, S. 3, http://www.zfl.gwzberlin.de/fileadmin/forschung/organisation/zfl_forschungsprogramm_2008_13.pdf, S. 3 (letzter Zugriff: 05.10.2011).

38 Wirth, Uwe, „Voruberlegungen zu einer Logik der Kulturforschung “, in: Ders. ( $\mathrm{Hg})$., Kulturwissenschaft. Eine Auswahl grundlegender Texte, Frankfurt a.M., 2008, S. 967, S. 66 . 